\title{
Scale-Free Networks: A Discrete Event Simulation Approach
}

\author{
Rex K. Kincaid ${ }^{1, \star}$ and Natalia Alexandrov ${ }^{2}$ \\ 1 The College of William and Mary, \\ Department of Mathematics, \\ Williamsburg, VA 23187-8795 \\ rrkinc@math.wm. edu \\ 2 NASA Langley Research Center, Hampton, VA 23681-2199 \\ n. alexandrov@nasa.gov
}

\begin{abstract}
This work is motivated by the need to reconsider the methods for the analysis and design of air transportation networks in order to meet increasing demands in the face of the current hub-and-spoke network near-saturation. In the late 1990s a number of researchers noticed that networks in biology, sociology, and telecommunications exhibited similar characteristics unlike traditional random networks. Three properties - small-world, power law, and constant clustering coefficientdescribe what are now most commonly referred to as scale-free networks. How do scale-free networks form? It is well documented that a network generated by adding nodes and edges preferentially will be scale-free. Are there other mechanisms? Why do networks organize themselves in this way? What causes a scale-free network to degrade? The focus of our research is to understand what drives a collection of nodes to organize as a scale-free network. Furthermore, once a network is scale-free what disrupts this apparently natural structure. To answer these questions we build a discrete-event simulation, nominally of an air transport system. The simulation is written in $\mathrm{C}$.
\end{abstract}

\section{Introduction}

The approaching saturation of the existing U.S. hub-and-spoke air transportation system is the source of growing interest in methods for the analysis and design of transportation architectures. The ambitious goals for the development of the next generation air transportation system set out by the Joint Planning and Development Office 1 require re-thinking about the nature of dynamic networks that have evolved over time rather than appeared through a centralized, planned design activity. The hub-and-spoke air transportation system is a scalefree network of a certain kind [7,6]; hence our interest in the nature of scale-free networks.

\footnotetext{
* The first author gratefully acknowledges the support of the NASA-NFFP program.

${ }^{1}$ http://www.jpdo.aero/site_content/index.html
} 
How and why do scale-free networks form? What causes a scale-free network to degrade? Part of the difficulty in answering these questions is limited understanding of the essential properties of scale-free networks. The relatively young literature (nearly all of the references are in the last seven years) on scale-free networks has primarily focused on three properties - average shortest path lengths, cumulative degree distributions, and clustering coefficients - to define these networks. However, it is as yet unclear whether or not these are the necessary and sufficient ingredients or are merely byproducts of some other unknown mechanisms.

Given the ubiquity of scale-free networks in nature and technology, as well as their desirable and undesirable properties, one would like to know whether, in some sense, this is the best possible structure a network can attain. If so, this has profound implications for the present (nearly) scale-free structure of air transportation networks: does the small number of hops between any pair of airports and clustering that lead to high connectivity and robustness to random attacks necessarily entail vulnerability to targeted attacks? Can one preserve the desirable properties of scale-free networks without the drawbacks? How can one increase the scalability of the current system in terms of its expandability? These are just a few questions we are attempting to explore in simplified networks in anticipation of dealing eventually with more realistic transportation systems.

Several books have been dedicated to the topic of scale-free networks, including the popular books Linked by Barabási [4 and Six Degrees by Watts [14. In addition, a number of excellent review articles exist including Newman [11, Strogatz [13], Albert and Barabási [1] and Dorogovtsev and Mendes [5]. These review articles contain hundreds of references. We note, as have many other authors, that networks whose cumulative degree distributions follow a power law are not new. One early example is Milgram's [10] work with acquaintance networks in the United States which led to his conclusion there were six degrees of separation among the individuals studied. However, the early references to networks following power laws are small in number when compared to the current explosion in interest.

In the next section we describe key properties of scale-free networks as well as computational mechanisms that can be used to generate two types of cumulative degree distributions observed in real networks. Section three examines the structure of simulated networks for a generic air transport systems. Section four provides results for a mechanism that degrades the power law of the degree distribution for scale-free networks.

\section{Properties of Scale-Free Networks}

Table 1 provides a summary of essential characteristics of four real networks. The interested reader is referred to Newman [11 for a more exhaustive table. The column labeled $C^{(1)}$ records one type of clustering coefficient - the number distinct paths of length two in triangles (three times the number of triangles) divided by the total number of distinct paths of length two in the network. For random networks clustering coefficients are known to approach zero as the 
Table 1. Basic statistics for four published Networks

\begin{tabular}{|c|c|c|c|c|c|}
\hline Networks & Nodes & Edges & AvgDeg & AvgSPD & $C^{(1)}$ \\
\hline protein & 2,115 & 2,240 & 2.12 & 6.80 & 0.072 \\
Internet & 10,697 & 31,992 & 5.98 & 3.31 & 0.035 \\
film actor & 449,913 & $25,516,482$ & 113.443 & 3.48 & 0.20 \\
Altavista & $203,549,046$ & $2,130,000,000$ & 10.46 & 16.18 & - \\
\hline
\end{tabular}

number of nodes grows large. The expected $C^{(1)}$ value if the film actor network were randomly constructed is .000252 . The Table 1 value of 0.20 is three orders of magnitude larger. Column 5 lists the average shortest path lengths for the networks in Table 1 . The value of 16.18 for the Altavista world wide web network means that Altavista web pages are on average 16 clicks away even though there are potentially 2.1 billion edges to traverse. If the average shortest path length scales logarithmically or slower with the number of nodes in the network then it is called a small-world network (see Watts [14). The natural logarithms of the number of nodes for the networks listed in Table 1 are 7.66, 9.28, 13.02 and 19.13 respectively. Consequently, all networks listed in Table 1 exhibit the small-world property. However, this property alone does not capture the essence of scale-free networks as traditional random networks are also small-world. The average degree of each network is recorded in column 4. The average degree can be used to estimate the clustering coefficient when the number of nodes is large.

The tail of the cumulative degree distributions for a scale-free network follows a power law. This is easiest to see if $P(k)$ versus $k$ is plotted on a log-log scale. If the tail follows a power law the graph of the tail will be a line on a log-log scale and the slope of the line is the exponent of the power function. If instead the tail follows an exponential law then a log-linear plot will be nearly linear. Both types of behavior have been observed in real networks. Networks grown by adding nodes and edges via preferential attachment are known to follow a power law. Researchers [12] have noted, however, that preferential attachment does not adequately explain large clustering coefficients.

Why do the degree distributions of some networks follow an exponential law rather than a power law? A network grown by adding nodes one at a time with edges placed between the new node and existing nodes by selecting the nodes closest, with respect to Euclidean distance, to the new node. Networks formed is this way are shown in 9 to follow an exponential law. Consequently, one partial answer to the above question is that the actual distances between nodes (not just the number of edges) play a critical role in the formation of exponential tails. A real network with an exponential tail is the power grid of the Western United States (see [11] page 187).

\section{Simulated Networks for an Air Transport System}

Air transportation networks have been analyzed in [7] and [6]. These authors show that the world-wide air transport system is a scale-free network although 
the cumulative degree distribution follows a truncated power law. It is speculated that the truncated effects are due to capacity restrictions at the airports. The network modeled in [7] and [6] consists of 3,883 cities (airports in the same city are aggregated) and 27,051 distinct city pairs having non-stop connections. One might have expected the cumulative degree distribution to have an exponential tail as did the U.S. power grid since the distance traveled by aircraft would seem to be a critical component. In fact, prior to the deregulation of the U.S. air transport system in 1978 air fares were closely tied to the distance traveled (see [3]). After deregulation fares for short-haul flights increased while denser markets for long-haul fares dramatically decreased. Consequently, distance traveled is no longer the primary mover in the U.S. air transport network. In contrast, Amaral et al. 2 analyze a network of world airports and conclude that the cumulative degree distribution follows an exponential distribution. However, the authors of [2] did not have access to the number of distinct flights between cities but rather the number of passengers in transit through each airport. The number of airports examined in [2] is not given.

The goal of our discrete event simulation is to answer two questions. What gross topological features trigger a set of nodes to organize as a scale-free network? What causes a scale-free network to degrade to some other network structure? We are not concerned with modeling the precise operational details of aircraft routing and scheduling (see 8] ) but rather the gross level network structure and how it arises.

Our simulation begins with a set of randomly generated points on a 5000 by 5000 unit square. Each point is assigned a weight generated from a statistical distribution. We think of the points as cities and the weights as relative population measures. We consider the entities that drive the simulation to be indistinguishable aircraft. The computational results reported here model 1000 cities and 2000 aircraft. Initially each aircraft selects one of the cities as an origination node $(\mathrm{O})$ and one as a destination $(\mathrm{D})$ node. If the Euclidean distance between $\mathrm{O}$ and $\mathrm{D}$ is larger than some user specified threshold then the flight route must go through an intermediate city ( $\mathrm{H}$ for hub). $\mathrm{H}$ is selected from a set of candidate cities as the one with minimum total weighted travel distance $(\mathrm{O}$ to $\mathrm{H}$ to $\mathrm{D}$ ) for all combinations of $\mathrm{H}$ with $\mathrm{O}$ and $\mathrm{D}$. Each plane is assigned a normally distributed speed with mean 400 and a standard deviation of 20 . The travel time is the travel distance divided by the speed. In addition there is a fixed time parameter that can be set to add a constant to each route to reflect time on the ground. When a simulated flight is completed a new O/D pair is selected. The simulation begins by scheduling an arrival for each of the 2000 planes.

During the simulation three pieces of data are collected. The number of times a given city is selected as an $\mathrm{O}, \mathrm{H}$, or $\mathrm{D}$ (called the hit distribution) and the number of times a flight leg is traversed between each possible pair of cities. If there are $n$ cities then there $n(n-1) / 2$ possible flight legs. Lastly, the number of times each city is a part of a distinct flight leg is recorded (called the degree distribution). Upon completion of the simulation a log-log plot of both the cumu- 
lative degree and hit distributions are plotted. If the tails of either (or both) are roughly linear then there is evidence that the underlying network is scale-free. If neither of the cumulative distributions follow a power law then there is no need to compute the average hop length and clustering coefficient. Statistics are not collected until after an initial warmup period to avoid the transient startup behavior.

Each simulation must specify the population distribution for the cities and the placement of the cities on the square grid. We experimented with two grid placements for the cities. The first follows a uniform distribution and the second follows a beta distribution. Sampling from $\operatorname{Beta}(0.5,0.5)$ for the $x$ and $y$ coordinates of each city pushes the cities closer to the boundaries of the square region imitating the clustering effects of cities near coastal boundaries (see Figure 1a). Several different population distributions were tested. For example, the 1000 cities were divided into three distinct population sizes-small, medium and large. Fifty-six percent of the cities were small with populations drawn from a normal distribution with a mean of 200 and a standard deviation of 5. Thirtytwo percent of the cities were medium with populations drawn from a normal distribution with a mean of 600 and a standard deviation of 20. Lastly, twelve percent of the cities were large with populations drawn from a normal distribution with a mean of 1800 and a standard deviation of 80 . The simulation selects origin and destination nodes preferentially based upon population size. Once the origin is selected candidate nodes for the destination must meet a user specified minimum distance threshold. Hubs were not part of these simulation experiments. Consequently, all routes are point to point. We found no examples in which the cumulative degree or hit distributions followed a power law. Further experimentation resulted in the assignment of a fitness value to each city. Initially all cities have a fitness of 1.0. Each time a city is selected as O or D its fitness is increased by 10 percent. After a certain amount of simulated time the fitness values follow a power law.

Next, we began a new series of simulation experiments by assigning fitness values following a power to each city as part of the initialization procedure. In addition the 100 most fit cities (out of 1000) were identified and set aside as potential hubs. During the simulation routes are selected point to point if the distance between origin and destination is less than a user specified threshold (recall that $\mathrm{O} / \mathrm{D}$ pairs already must meet a minimum distance threshold). If the $\mathrm{O} / \mathrm{D}$ distance is larger than the threshold then the route must travel through one of the 100 candidate hubs. The hub candidate list is further restricted by removing any hub cities that are too close to either the origin or the destination. Among the remaining hubs, the city that minimizes the O-H-D route distance weighted by $(1+\log$ (hub fitness $))^{-1}$ is selected. The origin is selected as before, preferentially based on population, but the destination selection includes the fitness information. That is, destinations are selected preferentially based on the fitness weighted population values. For all population distributions tested the resulting cumulative degree and hit distributions followed power laws. For example, if the population of every city is normally distributed with a mean of 
400 and a standard deviation of 20, the city locations are uniformly distributed, and all routes are point to point (no hubs) then the resulting cumulative degree distributions follows a truncated power law and the hit distribution follows a power law.

As we add the more realistic small, medium and large population center distributions, the beta distributed city locations, and allow hubs to part of the route structure both the cumulative degree distribution and hit distribution follow power laws. Clearly the power law distribution of the fitness values is the key to observing power law behavior for the cumulative degree and hit distributions. There are a variety of interpretations of these fitness values. For example, highly fit cities may be viewed as popular final destinations, or popular jump off points for cheaper fares to other locations.

The next two figures capture several key features of the simulated scale-free network. Due to space limitations we provide only one example. Figures 1(a) and $1(\mathrm{~b})$ are associated with output from the simulation model in which the power law fitness values are input values. The $x$ and $y$ coordinates of the cities are generated with $\operatorname{Beta}(0.5,0.5) * 5000.0$ and the cities population values follow the normally distributed small, medium and large values discussed earlier. Hubs are allowed to form and roughly $72 \%$ of the routes chosen make use of a hub. The remaining routes are point to point. Both hubs and destinations take into account fitness information in the selection process while the origins are selected preferentially based on population. About 68,000 flight routes are simulated in one run.

Figure 1(a) shows the geographical locations of each of the 1000 cities. Notice the clustering near the boundaries due to the beta distribution. The green triangles are placed at cities whose degree values are small. The blue circles are placed at cities whose degree values are average while the 15 red squares are placed at cities whose degree values are quite large. The red square labeled with

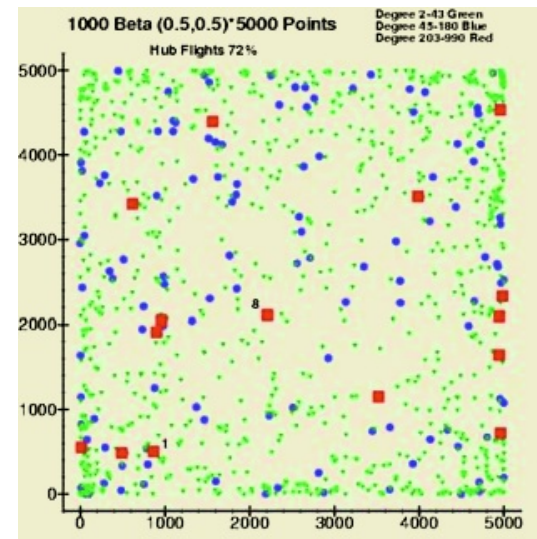

(a)

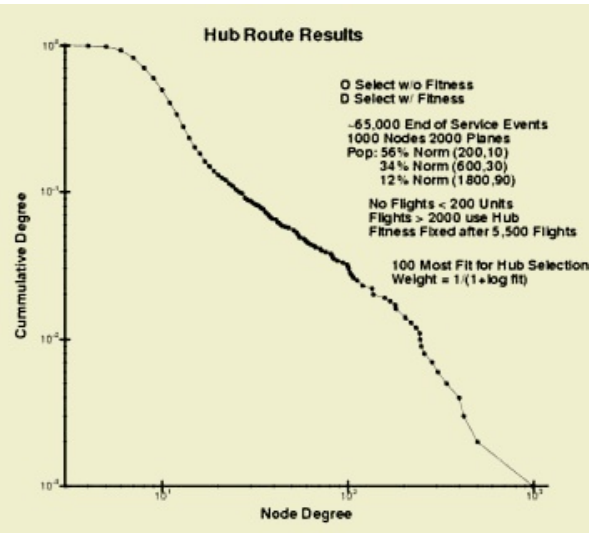

(b)

Fig. 1. 1000 cities - (a) Spatial degree dist.; (b)cummulative degree dist 
a 1 in figure 1(a) is the largest degree node while the red square labeled with an 8 has the eighth largest degree. If figure 1(a) were plotted for the hit distribution the red square with an 8 would have a 2 . That is, the eighth largest degree node had the second largest number of flights routed through it. This observation is in the same spirit as found in [7] where it was observed that the highest degree nodes were not necessarily the most critical for maintaining the network structure. Figure 1(b) displays the cumulative degree distribution on a log-log scale. It is easy to see that the tail of the plot is fit well by a linear function. The log-log plot of the cumulative hit distribution follows a similar pattern.

\section{Degradation of Power Laws}

The last question we address is what causes the power laws associated with a scale-free network to degrade. The authors of [7], 6] and [2] suggest that the scale-free structure of air transport networks is curtailed, either by a truncation of the power law or a switch to an exponential tail, when capacity restrictions ensue. The capacity restrictions might take the form of fewer flights along existing routes (changing the number of hits but not the degree) thereby forcing overflow demand to be routed to other nearby cities, or by limiting the number of routes that can be flown into the airport. One approach to capture this effect in a simulation is to model each of the cities as a finite capacity queue and develop rules for selecting hubs and destinations based on the status of the queues. In an attempt to keep things as simple as possible in our simulation we have avoided queues. Instead we allow each city to keep a short list (of length three in our

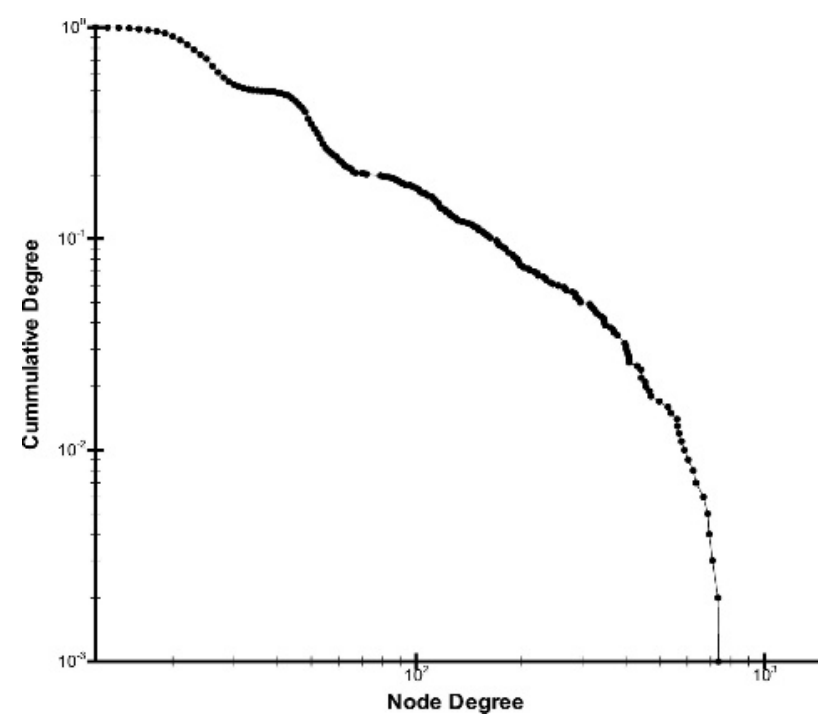

Fig. 2. Degree distribution: local capacity restrictions 
example) of the most recent events (arrivals and departures). When considering selection of hubs and destination nodes if the time between the last event time on the city's list and the current simulation time is too small then the node is removed from consideration for selection. The cumulative degree distribution for this simulation run is displayed in figure 2. The truncated effect is easily seen when compared to figure $1(\mathrm{~b})$.

\section{References}

1. Albert, R. and A.-L. Barabási, "Statistical Mechanics of Complex Networks," Rev. Modern Physics, 74:42-47 (2002).

2. Amaral, L.A.N., A. Scala, M. Barthelemy and H.E. Stanley, "Classes of small-world networks," Proc. Natl. Acad. Sci. USA, 97:11149-11152 (2000).

3. Bailey, E.E., D.R. Graham, and D.P. Kaplan, Deregulating the Airlines, MIT Press, 1985.

4. Barabási, A.-L., Linked: The New Science of Networks, Perseus, Cambridge, MA, 2002

5. Dorogovtsev, S.N. and J.F.F. Mendes, "Evolution of Networks," Advances in Phys., 51:1079-1187 (2002).

6. Guimera, R., S. Mossa, A. Turtschi and L.A.N. Amaral, "Structure and Efficiency of the World-Wide Airport Network," Preprint 0312535 (2003) available from http://arxiv.org/abs/cond-mat/.

7. Guimera, R. and L.A.N. Amaral, "Modeling the world-wide airport network," European Physical Journal B, 38, 381-385 (2004).

8. Johnson, E., J. Banks, L.H. Lee, H.C. Huang, C. Lee, E.P. Chew, W. Jaruphongsa, Y.Y. Yong, Z. Liang, C.H. Leong, Y.P. Tan, and K. Namburi, "Discrete Event Simulation Model for Airline Operations: SIMAIR," Proceedings of the 2003 Winter Simulation Conference, S. Chick, P.J. Sanchez, D. Ferrin, and D.J. Morrice (editors), pp. 1656-1662.

9. Kincaid, R.K., "Scale-free Graphs for General Aviation Flight Schedules," NASA technical memorandum (2003) November, NASA/CR-2003-212648. (http://techreports.larc.nasa.gov/ltrs/ltrs.html)

10. Milgram, S., Psych. Today, 2:60 (1967).

11. Newman, M.E.J., "The Structure and Function of Complex Networks," SIAM Review, 45:167-256 (2003).

12. Solé, R.V., R. Ferrier-Cancho, J. Montoya and S. Valverde, "Selection, Tinkering and Emergence in Complex Networks," Complexity, 8:1-32 (2003).

13. Strogatz, S.H., "Exploring Complex Networks," Nature, 410:268-276, March (2001).

14. Watts, D.J., Six Degrees: The Science of a Connected Age, Norton, New York, 2003. 